\title{
Active Equalization for Lithium Batteries Combined Cell Voltage with State of Charge
}

\author{
Qinghe Liu, Tao Yang, Qingyang Xu, Lijun Zhao \\ School of Automotive Engineering, Harbin Institute of Technology at Weihai, Weihai, China \\ yangtao130@126.com
}

\begin{abstract}
The equalization strategy simply based on cell voltage or state of charge is used in pure electric racing car. However, there is deviation in estimating state of charge or detecting cell voltage, which causes switches to open and close frequently or improperly, lowing equalization efficiency. Taking energy utilization and equalization efficiency into account, according to the actual operating characteristics of lithium batteries, we studied one new equalization strategy combined cell voltage and state of charge. First of all, one kind of lithium battery model was built and then the active equalization circuit based on the battery model was also built. Secondly, the equalization strategy was simulated. At last, one discharge experiment on the equalization module was conducted. The simulation and experiment results show that our strategy can improve energy utilization, reduce opening and closing times of switches and obtain better equalization effects.
\end{abstract}

Keywords-pure electric racing car; battery consistency; battery model; equalization strategy

\section{INTRODUCTION}

Pure electric racing cars are becoming popular in various domestic competitions. Lithium batteries are series connection in pure electric racing cars, and the battery has different characteristics in terms of initial capacity, resistance and self-discharge ratio ${ }^{[1]}$. The inconsistency of batteries has a serious impact on the performance of battery packs ${ }^{[2]}$. To solve this problem, on the one hand, batteries are sorted before usage by test and those with good consistency are selected $^{[3]}$. On the other hand, the inconsistency will be limited within a reasonable range through appropriate equalization technology. At present, the study on equalization technology is focused on equalization strategy and equalization structure. The effect is not good when the related research applied to complex driving conditions.

Equalization strategy focuses on equalization standards and control methods. Reference [4] used voltage as equalization standards to judge inconsistency of batteries and verified working effects of the equalization on improvement of inconsistency under different conditions, such as shelving, charge and discharge; Reference [5] used state of charge (SoC) as equalization standards to amend the actual capacity of the battery; Reference [6] used voltage or SoC respectively as equalization standards to verify the equalization effects of same batteries. For equalization methods, in Reference [7] the equalization current was controlled based both on fuzzy control theory and the current voltage. Reference [8] used Simulink to build equalization model, simulated and verified the control algorithm by using SoC as equalization standard. Reference [9] proposed one equalization method based on different modes of control strategies. Equalization structures are multiple. Reference [10] proposed one distributed active equalization system based on inductance; Reference [11] proposed one active equalization solution based on transformer to optimize the duty ratio; Reference [12] equipped with an equalization unit for each battery, but the structure was complex and the practicality was not good.

This paper used the combination of voltage and state of charge as equalization standards, built equivalent circuit model for lithium batteries and equalization structure based on the battery model. The equalization strategy was applied to the equalization structure. The simulation and experiment were conducted to prove that our strategy can improve energy utilization and avoid the switches opening and closing frequently.

\section{EQUALIZATION STRATEGY FOR BATTERY PACK}

\section{A. Equalization Standard}

We conduct the discharge experiment for pure electric racing car by using 16Ah lithium battery. The characteristic curve of cell voltage during discharge is shown in Fig. 1. The figure shows that the cell voltage and SoC are positive correlation. We define it as plateau when SoC is in a range of $20 \% \sim 80 \%$ and the cell voltage changes gently. Outside of the plateau, voltage changes drastically and easy to fluctuate.

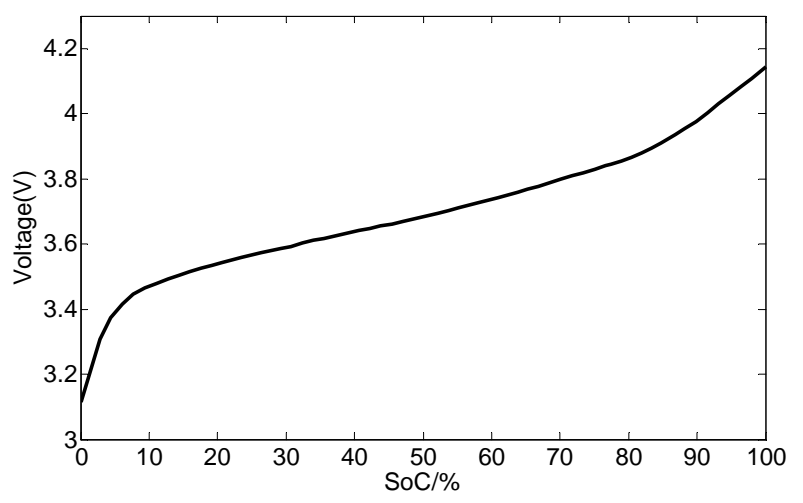

Figure 1. Characteristic curve of cell voltage during discharge.

At the beginning or end of discharge, the cell voltage is sensitive to the changes of $\mathrm{SoC}$ and has measurement deviation. If the equalization strategy is based on voltage, it 
is likely to cause the switches to open and close frequently, increasing energy consumption. To make matters worse, the battery maybe overcharge or over-discharge. Therefore, we select SoC as the equalization standard at this stage and decrease equalization threshold. On the contrary, the voltage is not sensitive to the change of SoC at the plateau and the voltage is easy to measure, so we select voltage as equalization standard and increase the equalization threshold at this stage.

\section{B. Equalization Threshold}

We select SoC as equalization standard outside of plateau. In order to confirm the equalization threshold, we define two equations as follows

$$
\left\{\begin{array}{l}
\delta q_{n}=\left|q_{n+1}-q_{n}\right| \\
\Delta q=h(\mathrm{SoC}) \cdot q_{0}
\end{array} .\right.
$$

Where $\delta q_{n}$,called difference value of SoC between two adjacent batteries at any time; $q_{n}$, represents the SoC of any battery, $\mathrm{n}=(1,2,3, \ldots) ; \Delta q$, called equalization threshold of the battery pack under the current SoC; $h$ (SoC),called threshold tendency function, SoC represents the current remaining capacity of the battery pack; $q_{0}$,represents the minimum equalization unit.

We know that the closer to the cut-off voltage the more likely to lead to overcharge or over-discharge, so we use threshold tendency function $h$ (SoC) to make equalization threshold smaller when it closes to the cut-off voltage. The $q_{0}$ is set to $1 \%, h(0)=h(100 \%)=0.3, h(20 \%)=h(80 \%)=3$. When SoC varies from 0 to $20 \%, h(\mathrm{SoC})$ is linearly increased and when SoC varies from 80 to $100 \%, h(\mathrm{SoC})$ is linearly decreased. If the difference value of SoC between two adjacent batteries reaches equalization threshold, the process of equalization will start.

We select voltage as equalization standard at the plateau. We define one equation

$$
r=\left|v_{i}-v_{i+1}\right|, i=(1,2,3, \ldots)
$$

Where $r$, represents difference value of voltage between two adjacent batteries at any time; $v_{i}$, represents cell voltage. The $r$ is defined as a constant at the plateau because the voltage changes smoothly. When $r=25 \mathrm{mV}$, the equalization process will start.

\section{Equalization Strategy}

Cell voltage is detected and SoC is estimated at any moment in the process of charge and discharge. Equalization will start when the difference value of SoC between two adjacent batteries reaches equalization threshold outside of the plateau. Energy with high capacity battery will be transferred to the low one. The case can be done in the same manner when the battery is in the plateau. After these two stages, the voltage or the SoC of adjacent batteries are controlled within the threshold in the process of charge or discharge. The battery pack can be filled or released more energy and energy utilization will be improved.

The flow in Fig. 2 shows the process of equalization.

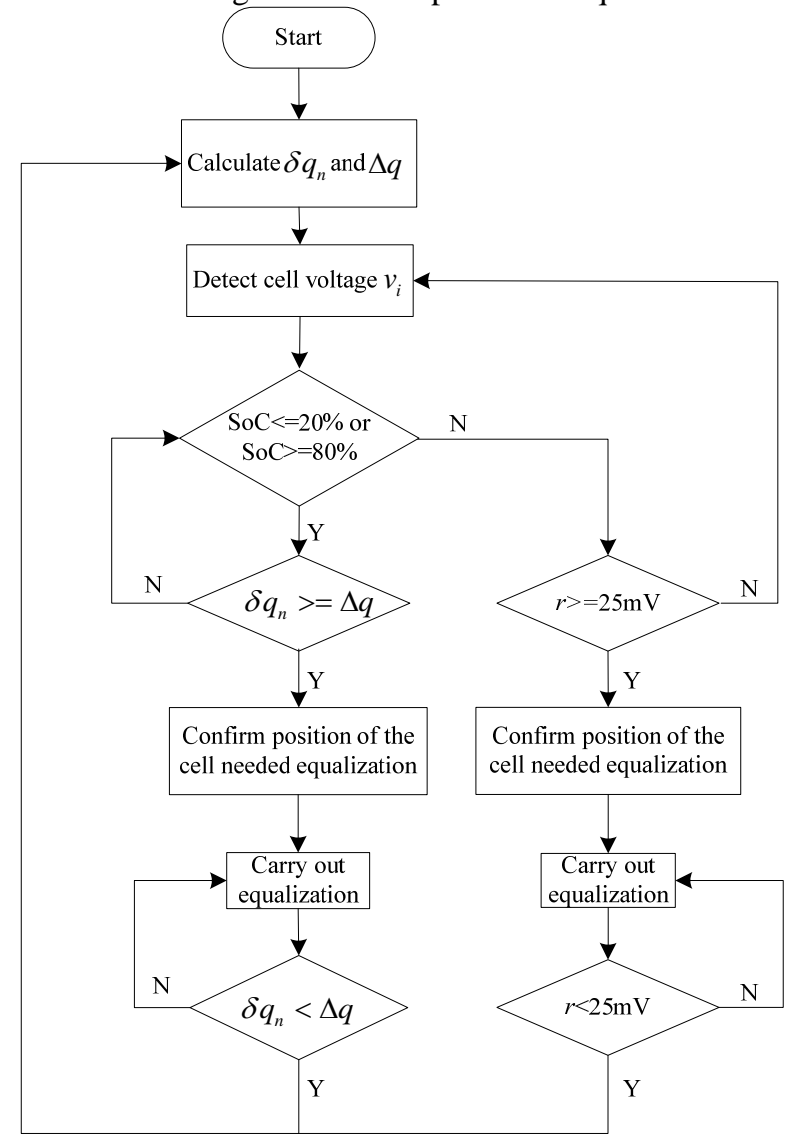

Figure 2. Flow chart of equalization control.

\section{BATTERY MODEL BUILDING AND SIMULATION OF EQUALIZATION STRATEGY}

\section{A. Battery Model Building}

With the rebound voltage and the hysteresis voltage of the battery been considered, the battery model can be equivalent to one equivalent voltage source and a series of equivalent impedance. The non-linear relation between hysteresis voltage $\mathrm{V}_{h}$ and Electro-Motive Force(EMF) can be expressed as controlled source. The equivalent impedance model can be expressed by the third order RC network. The equivalent circuit model is shown in Fig. 3. 


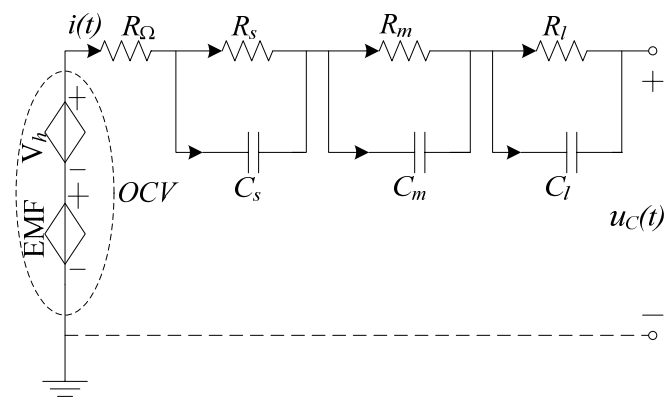

Figure 3. equivalent circuit model for lithium battery.

The characteristic of rebound voltage can be described by the equivalent impedance model. According to the experimental data, we adopt (3) to fit the curve of rebound voltage and obtain the parameter values of the model.

$$
\begin{gathered}
u_{C}(t)=O C V-i(t) R_{\Omega}-i(t) R_{s} e^{-\frac{t}{R_{s} C_{s}}}-i(t) R_{m} e^{\frac{t}{R_{m} C_{m}}} \\
-i(t) R_{l} e^{-\frac{t}{R_{l} C_{l}}}
\end{gathered}
$$

Where $u_{C}(t)$,represents cell voltage at any time; $R_{\Omega}$,represents ohmic resistance; $R_{s}, R_{m}, R_{l}$, represent the resistance values of the third order RC network; $C_{s}, C_{m}$, $C_{l}$,represent the capacitance values of the third order RC network; $i(t)$ represents current at any time.

We adopt Simulink to build the equivalent circuit model of the battery. The simulation structure of battery model is shown in Fig. 4.

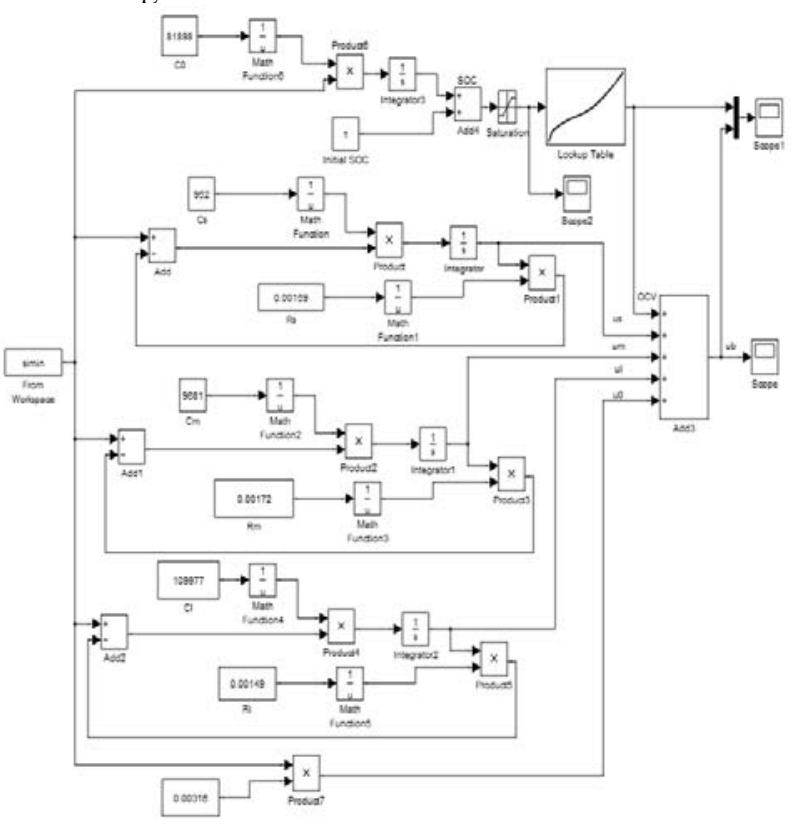

Figure 4. Simulation structure of battery model.

\section{B. Simulation of Equalization Strategy Based on Battery Model}

The battery model can be applied to an equalization structure with many batteries in series. Taking three adjacent batteries as an example, we can make the energy transferred between the adjacent batteries by controlling the MOSFET. If the difference value of voltage or SoC between adjacent batteries below the threshold, the process of equalization stops. Simulation structure of inductance-based equalization is shown in Fig. 5.

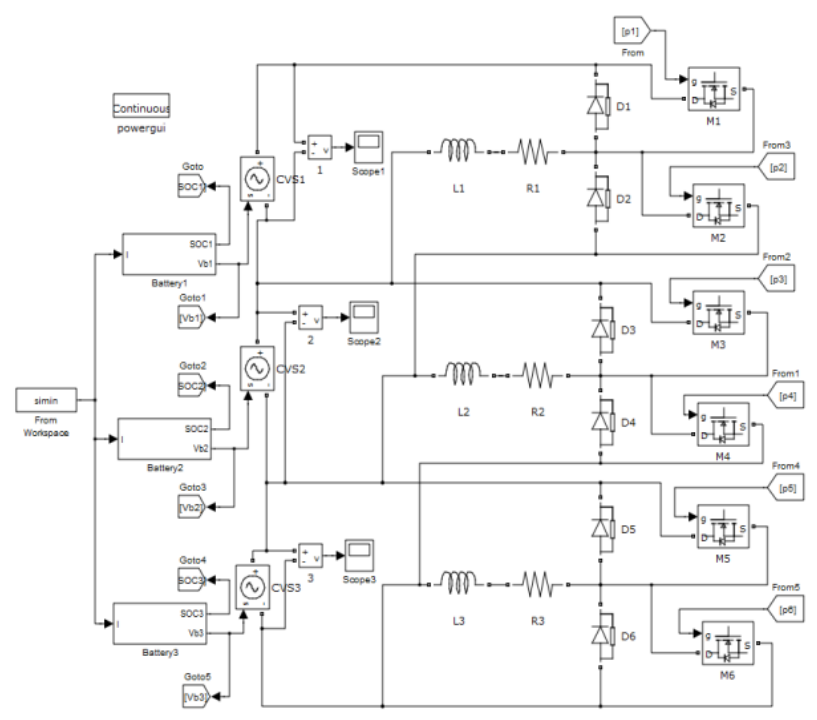

Figure 5. Simulation structure of inductance-based equalization.

Outside the plateau, we set three batteries' initial SoC of 0.92, 0.90, 0.88. The simulation result is shown in Fig. 6. It is shown that, at the end of the equalization, the difference values of SoC between adjacent batteries are within $0.5 \%$, which indicates better equalization performance.

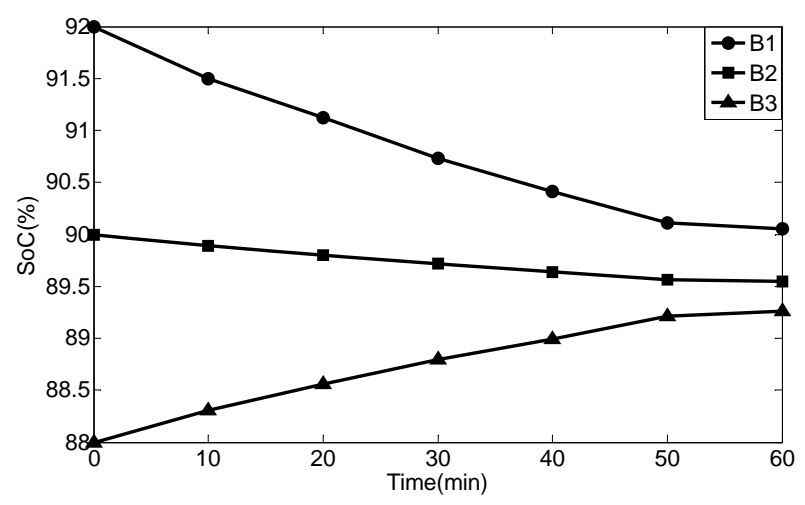

Figure 6. Equalization simulation based on SoC.

In the plateau, we set three batteries' initial voltage of $3.65 \mathrm{~V}, 3.69 \mathrm{~V}$ and $3.72 \mathrm{~V}$. The simulation result is shown in Fig. 7. It is shown that, at the end of the equalization, the difference values of voltage between adjacent batteries are within $8 \mathrm{mV}$, which also indicates better equalization performance. 


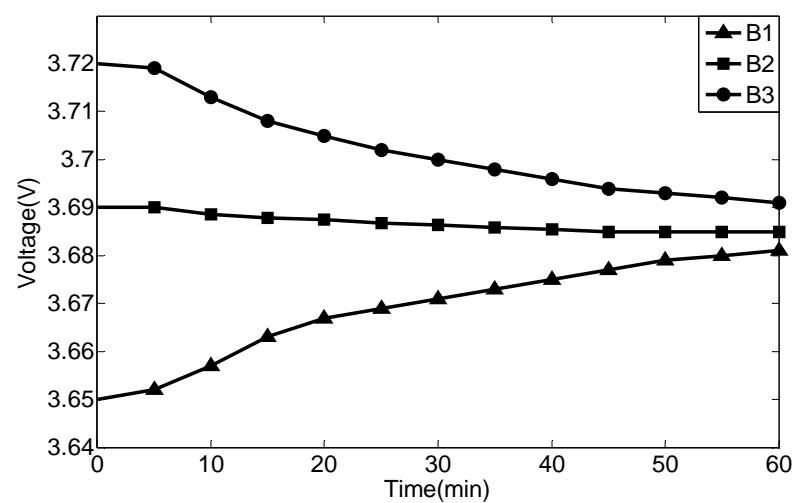

Figure 7. Equalization simulation based on voltage.

\section{EQUALIZATION EXPERIMENT}

We use five batteries of 16Ah, called B1, B2, B3, B4, B5, as experimental samples. The experiment is conducted under the constant temperature of $25^{\circ} \mathrm{C}$. The batteries' capacity should be calibrated at first before the experiment, after that the battery was fully charged.

To examine the effect of the equalization, the experiment was divided into four groups: (1) no equalization, (2) equalization based on the cell voltage, (3) equalization based on the SoC, and (4) equalization combined voltage with SoC. In the experiment, if any one battery reaches the cut-off voltage $(3.2 \mathrm{~V})$, the process of discharge will stop immediately. The voltage distribution and the released electric energy of the battery pack were recorded at the end of discharge. Then each cell discharges individually and the total electric energy was recorded. During the experiment, opening and closing times of the switches were also recorded. Considering the complicated driving conditions of electric racing car, we use the Urban Dynamometer Driving Schedule (UDDS) to test the effect of equalization.

The voltage comparison of four methods at the end of discharge is shown in Fig. 8. We can see that the voltage distribution of the fourth group is closer to cut-off voltage at the end of discharge. It shows that this method can effectively prevent batteries from over-discharge.

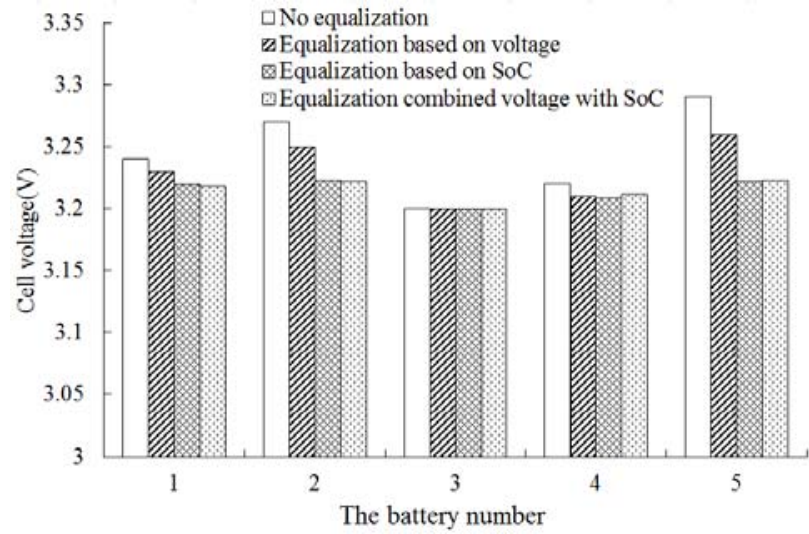

Figure 8. The voltage comparison of four methods at the end of discharge.
The state of charge comparison of four methods at the end of discharge is shown in Fig. 9. The sum of SoC of four methods after discharge respectively is $11.7 \%$, $7.8 \%, 5.3 \%$ and $4.9 \%$ of the total capacity. The results show that the battery has higher energy utilization by adopting the fourth method.

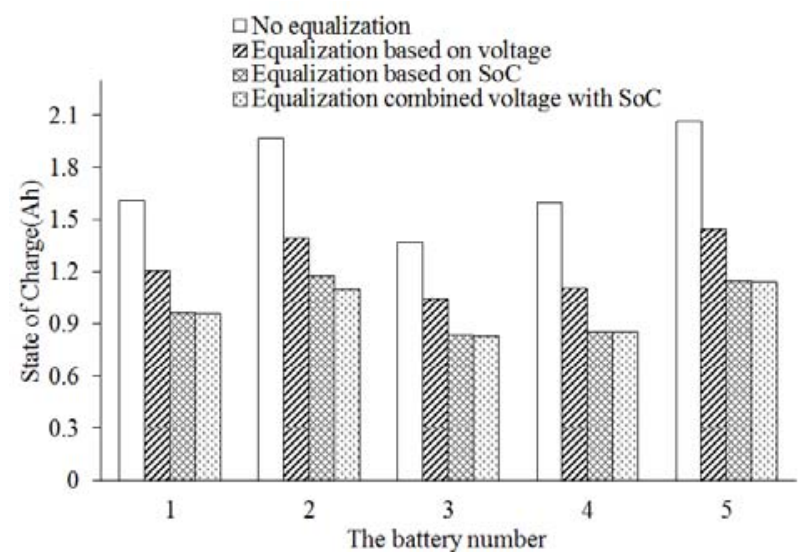

Figure 9. The state of charge comparison of four methods at the end of discharge.

Comparison of opening and closing times during discharge is shown in Table 1.There is only 12 opening and closing times of switches during discharge by using our equalization method. Therefore, it has better effect in avoiding switches opening and closing frequently and higher efficiency of equalization.

TABLE I. COMPARISON OF OPENING AND CLOSING TIMES DURING DISCHARGE

\begin{tabular}{|c|c|}
\hline Methods of Equalization & $\begin{array}{c}\text { opening and closing } \\
\text { times of switches } \\
\text { (times/hour) }\end{array}$ \\
\hline Equalization based on cell voltage & 97 \\
\hline Equalization based on SoC & 23 \\
\hline $\begin{array}{c}\text { Equalization combined cell voltage } \\
\text { with SoC }\end{array}$ & 12 \\
\hline
\end{tabular}

\section{CONCLUSIONS}

In this paper, one kind of equalization strategy combined cell voltage with SoC was proposed.

The battery model and equalization structure based on the battery model were built by using MATLAB/Simulink. The equalization strategy was simulated. Results show that our strategy works well.

The equalization experiment was conducted by using the Urban Dynamometer Driving Schedule. The results show that our strategy has a better effect to avoid battery overcharge or over-discharge. Meanwhile, it also increases energy utilization and has better effect in avoiding switches opening and closing frequently.

Cycle index and aging degree of batteries should be taken into account in the future studies. The equalization 
threshold can be optimized to make equalization efficiency and energy utilization better.

\section{ACKNOWLEDGMENT}

The authors would like to thank Shandong Province Science and Technology Development Project 2014GGX105006 for financial support.

\section{REFERENCES}

[1] K. C. Xu, C. Q. Gui. Difference between Li-ion single battery and battery group[J]. Battery Bimonthly, 2011:315-318.

[2] Z. P. Wang, F. C. SUN, C. Lin. An Analysis on the Influence of Inconsistencies Upon the Service Life of Power Battery Packs[J]. Transactions of Beijing Institute of Technology, 2006, 26(7): 577-580.

[3] J. Kim, J. Shin, C. Chun. Stable Configuration of a Li-Ion Series Battery Pack Based on a Screening Process for Improved Voltage/SoC Equalization[J]. Power Electronics, IEEE Transactions on, 2012, 27(1):411 - 424.

[4] A. M. Imtiaz, F. H. Khan, H. Kamath. A low-cost time shared cell equalization technique for future lithium-ion battery storage system featuring regenerative energy distribution[C]. Applied Power Electronics Conference and Exposition (APEC), 2011 Twenty-Sixth Annual IEEE. IEEE, 2011:792 - 799.
[5] J. H. Han, L. F. Wang, Z. Y. Liu. Equalization strategy for lithium battery pack based on the state of charge[J]. Journal of Electronic Measurement and Instrumentation, 2014,(10).

[6] M. Einhorn, M. Roessler, J. Fleig. Improved Performance of Serially Connected Li-Ion Batteries With Active Cell Equalization in Electric Vehicles[J]. Vehicular Technology, IEEE Transactions on, 2011, 60:2448 - 2457.

[7] G. Q. Jiang, J. X. Zhu, Q. Z. Shen. Equalization system for serial lithium-ion battery string based on fuzzy logical control[J]. Electronic Technology, 2008,(7).

[8] M. Daowd, M. Omar, P. Van Den Bossche. Passive and active battery equalization comparison based on MATLAB simulation[C]. //Vehicle Power and Propulsion Conference, IEEE. IEEE, 2011:1 - 7.

[9] F. Pei, K. G. Zhao. Strategy of battery transient equilibrium control for electric vehicle[J]. Mechanical \& Electrical Engineering Technology, 2008, (8).

[10] H. F. Dai, X. Z. Wei, Z. C. Sun. Inductance-based active equalization of lithium-ion batteries for EV applications[J]. Journal of Tongji University(Natural Science), 2013, (10):1547-1553.

[11] W. Y. Zheng, S. J. Hu, Z. Niu. Research on active equalization scheme of power battery pack[J]. Journal of Electronic Measurement and Instrumentation, 2014, (7).

[12] D. Song, Y. H. Niu, L. R. Wang. Research on non-dissipative cell equalizer for space lithium-ion battery[J]. Spacecraft Engineering, 2014,23(4). 